Acta horticulturae et regiotecturae 1

Nitra, Slovaca Universitas Agriculturae Nitriae, 2014, p. 24-28

\title{
ROUTING OF VEGETABLE PRODUCTION IN THE NITRA REGION IN 2002-2010
}

\author{
Magdaléna VALŠíKOVÁ*, Rudolf RYBAN, Katarína SRNIČKOVÁ \\ Slovak University of Agriculture in Nitra, Slovak Republic
}

The paper deals with the evaluation of the development of cultivation area and production of vegetables in different districts of the Nitra region in the period of the years 2002-2010. The region of Nitra has excellent climatic conditions for the cultivation of agricultural crops, including vegetables. Among the most widely grown vegetables belong cabbage, carrots, parsley, onions, tomatoes, peppers, red watermelons, and sweet corn.

Keywords: vegetables, production, area

The Nitra region covers an area of $6,343 \mathrm{~km}^{2}$, occupying $12.9 \%$ of the area of Slovakia. In the region there are 350 municipalities, 15 of which have city status. The county is divided into seven districts, namely: Komárno, Levice, Nitra, Nové Zámky, Šal'a, Topol'čany, and Zlaté Moravce. The largest of them is the Levice district, and conversely the smallest one is the Šal'a district.

The land relief of the region is flat, punctuated by hills. The mountain range Tríbeč stretches to the north. The northeast part is bordered by the foothills of the Štiavnicke vrchy and the Pohronský Inovec. The southern and southeastern parts belong to the Danubian plain. The following rivers flow though the region: the Danube, the Váh, the Nitra, the Hron, the Žitava, and the Ipel.' There is good quality agricultural land, especially moderate loamy soils (Srničková, 2012). The region has excellent climatic conditions for the cultivation of agricultural crops, including vegetables (Uher, 2011).

\section{Material and methods}

To assess the production of vegetables in the Nitra region, we used available official data of the Statistical Office of the Slovak Republic from 2002 to 2010 (Rozborilová et al., 2010) and other sources and published papers (Jureková et al., 2005; Meravá et al., 2009). The data obtained were processed in tables and figures, by area (ha) and production of vegetable species ( $t$ ) in different districts during the reporting year (Valšíková et al., 2011). The tables are complemented by verbal commentaries. In the tables, the kinds of vegetables that are cultivated in small areas and their production are omitted. All data represent the management on arable land except of gardens.

\section{Results and discussion}

Acreage and production of vegetables in the Nitra region by districts in 2010 is given in Table 1. Based on this, it is clear that the vegetables are grown on the largest area of arable land (937 ha) in the district of Levice. Vegetables were planted approximately on the same area of 780 ha in the districts of Komárno and Nové Zámky. There was 593 ha of vegetables grown in the Nitra district (Meravá et al., 2011).

The greatest volume of vegetables was produced in the district of Levice $(7,343 \mathrm{t})$, followed by the Nové Zámky district $(7,114 \mathrm{t})$ and the district of Komárno $(4,538 \mathrm{t})$. The district of Šal'a produced 2,775 t, the district of Nitra 2,091 t of vegetables. The lowest volume of vegetables was produced in the districts of Topol'čany (123 t) and Zlaté Moravce (27 t). The percentage of participation on production according to the districts is given in Fig. 1.

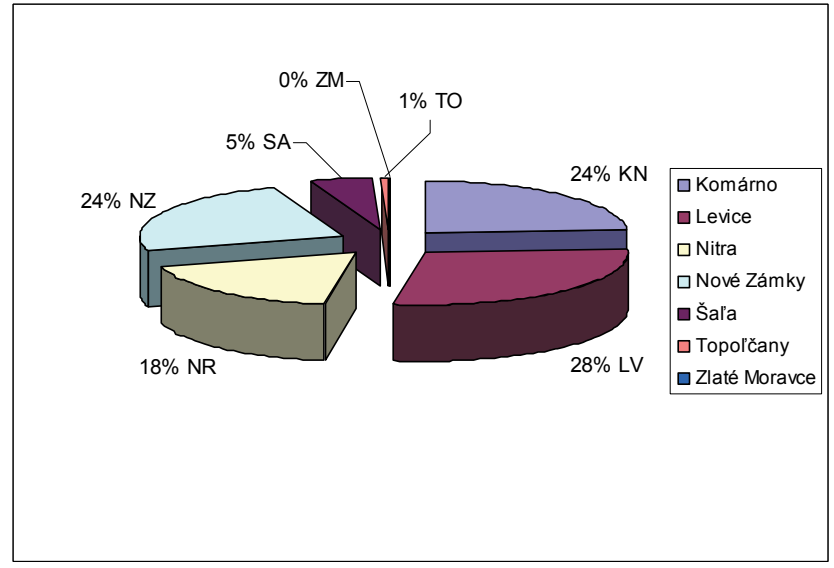

Figure 1 Participation of districts on the production of vegetables in the Nitra region in \%

The area of vegetables production

In Table 2 we evaluate the area of vegetable species in the years 2002-2010 in the district of Komárno. From this it can be seen that since 2002, the production area declined from 2,020 ha in 2002 to 782 ha in 2010. The lowest value was recorded for 2009 (Valšíková and Uher, 2009). Parsley with parsnips, onions, tomatoes, white cabbage and peppers were grown in 2002 on the largest area. For these and other species the growing area has reduced over the time (Valšíková et al., 2011).

In 2002, the district of Levice marked 1,606 ha of vegetables. From that amount, tomatoes were grown on 346 ha, corn on 269 ha, gherkins on 243 ha and beans on 
Table 1 Harvested area and production volume in the districts of the Nitra region in 2010

\begin{tabular}{|l||c|c|}
\hline Districts & Area of vegetables in ha & Production of vegetables in t \\
\hline \hline Komárno & 782.42 & 4,538 \\
\hline Levice & 937.31 & 7,343 \\
\hline Nitra & 593.51 & 2,091 \\
\hline Nové Zámky & 783.73 & 7,114 \\
\hline Šala & 161.71 & 2,775 \\
\hline Topoľčany & 16.82 & 123 \\
\hline Zlaté Moravce & 4.30 & 27 \\
\hline Total in the region of Nitra & $3,279.80$ & 24,012 \\
\hline
\end{tabular}

Table 2 Harvested area of most grown species of vegetables in the district of Komárno in ha

\begin{tabular}{|l||c|c|c|c|c|c|c|c|c|}
\hline Vegetables & $\mathbf{2 0 0 2}$ & $\mathbf{2 0 0 3}$ & $\mathbf{2 0 0 4}$ & $\mathbf{2 0 0 5}$ & $\mathbf{2 0 0 6}$ & $\mathbf{2 0 0 7}$ & $\mathbf{2 0 0 8}$ & $\mathbf{2 0 0 9}$ & $\mathbf{2 0 1 0}$ \\
\hline \hline White cabbage & 252.37 & 244.63 & 141.35 & 63.52 & 85.47 & 60.68 & 49.40 & 43.36 & 36.23 \\
\hline Cauliflower & 52.68 & 39.88 & 38.59 & 31.21 & 34.20 & 16.62 & 9.25 & 12.74 & 14.05 \\
\hline Savoy cabbage & 40.39 & 34.25 & 30.80 & 14.87 & 22.79 & 12.94 & 9.92 & 9.45 & 9.94 \\
\hline Kohlrabi & 41.61 & 47.95 & 37.95 & 29.26 & 35.79 & 23.84 & 10.62 & 15.52 & 17.20 \\
\hline Carrots & 180.61 & 108.59 & 126.24 & 65.73 & 69.08 & 80.18 & 54.31 & 71.44 & 39.71 \\
\hline Parsley and parsnips & 278.86 & 143.33 & 139.58 & 135.76 & 127.43 & 120.01 & 114.68 & 110.99 & 136.78 \\
\hline Cucumbers & 54.26 & 35.73 & 27.48 & 33.36 & 28.11 & 29.93 & 3.28 & 6.45 & 5.70 \\
\hline Watermelon & 145.85 & 168.71 & 137.76 & 117.74 & 73.08 & 67.75 & 53.97 & 41.78 & 28.10 \\
\hline Peppers & 216.07 & 223.33 & 183.00 & 177.92 & 151.48 & 127.08 & 72.42 & 79.95 & 72.14 \\
\hline Tomatoes & 261.37 & 240.48 & 228.63 & 260.94 & 240.73 & 230.87 & 90.15 & 109.42 & 87.43 \\
\hline Onion & 268.31 & 224.09 & 254.90 & 126.63 & 114.40 & 143.67 & 144.03 & 60.26 & 60.96 \\
\hline Head lettuces & 16.36 & 18.67 & 15.73 & 12.27 & 10.42 & 13.05 & 8.42 & 12.04 & 10.15 \\
\hline Peas & 5.04 & 3.59 & 43.15 & 2.86 & 2.86 & 1.04 & - & - & 51.61 \\
\hline Sweet corn & 13.90 & 11.31 & 12.37 & 7.96 & 8.17 & 4.10 & - & 11.70 & 31.90 \\
\hline Total & $2,020.00$ & $1,708.38$ & $1,598.00$ & 1211.64 & $1,161.70$ & $1,035.71$ & 705.04 & 700.46 & 782.42 \\
\hline
\end{tabular}

Table 3 Harvested area of most grown species of vegetables in the district of Levice in ha

\begin{tabular}{|l||c|c|c|c|c|c|c|c|c|}
\hline Vegetables & $\mathbf{2 0 0 2}$ & $\mathbf{2 0 0 3}$ & $\mathbf{2 0 0 4}$ & $\mathbf{2 0 0 5}$ & $\mathbf{2 0 0 6}$ & $\mathbf{2 0 0 7}$ & $\mathbf{2 0 0 8}$ & $\mathbf{2 0 0 9}$ & $\mathbf{2 0 1 0}$ \\
\hline White cabbage & 69.36 & 109.47 & - & 13.83 & 39.87 & 14.79 & - & 6.08 & 6.70 \\
\hline Carrots & 26.36 & 85.40 & 64.65 & 17.81 & 27.86 & 8.96 & 12.37 & 5.47 & 21.23 \\
\hline Parsley and parsnips & 27.69 & 12.90 & 17.18 & 9.68 & 19.13 & 9.38 & 9.92 & 5.35 & 29.75 \\
\hline Gherkins & 242.91 & 140.52 & 77.04 & 99.03 & 93.03 & 50.25 & 41.22 & 34.16 & 33.90 \\
\hline Cucumbers & 5.43 & 17.61 & 8.12 & 4.36 & 3.46 & 0.49 & 2.10 & 2.00 & 2.00 \\
\hline Peppers & 193.97 & 169.56 & 105.90 & 100.73 & 84.69 & 63.63 & 19.69 & 17.59 & 19.87 \\
\hline Tomatoes & 363.87 & 263.05 & 269.33 & 165.56 & 217.54 & 96.29 & 39.66 & 17.75 & 38.85 \\
\hline Onion & 89.20 & 60.79 & 108.77 & 60.90 & 59.70 & 28.98 & 55.74 & 42.68 & 57.64 \\
\hline Peas & 51.25 & 108.54 & 250.08 & 235.40 & 1.98 & - & - & - & 347.85 \\
\hline Sweet corn & 268.85 & 284.25 & 242.58 & 161.14 & 70.67 & 347.77 & 370.40 & 215.50 & 204.74 \\
\hline Pumkin & - & 62.00 & 30.89 & - & 66.51 & 56.29 & 55.03 & 72.80 & 73.86 \\
\hline Total & $1,606.04$ & $1,449.07$ & $1,313.48$ & 998.98 & 757.06 & 797.45 & 788.21 & 809.12 & 937.31 \\
\hline
\end{tabular}


Table 4 Harvested area of most grown species of vegetables in the district of Nitra in ha

\begin{tabular}{|l||c|c|c|c|c|c|c|c|}
\hline Vegetables & $\mathbf{2 0 0 3}$ & $\mathbf{2 0 0 4}$ & $\mathbf{2 0 0 5}$ & $\mathbf{2 0 0 6}$ & $\mathbf{2 0 0 7}$ & $\mathbf{2 0 0 8}$ & $\mathbf{2 0 0 9}$ & $\mathbf{2 0 1 0}$ \\
\hline \hline White cabbage & 55.00 & 75.70 & 38.04 & 39.38 & 30.96 & 48.25 & 23.38 & 47.30 \\
\hline Cauliflower & 14.02 & 13.24 & 13.32 & 24.08 & 12.65 & 9.50 & 8.58 & 7.30 \\
\hline Carrots & 4.99 & 4.60 & 3.72 & 3.82 & 2.69 & 21.12 & 7.60 & 7.60 \\
\hline Gherkins & 19.60 & 20.33 & 23.59 & 28.32 & 15.54 & 14.40 & 15.60 & 15.27 \\
\hline Cucumbers & 2.37 & 2.63 & 2.34 & 2.59 & 2.14 & 5.00 & 5.00 & 5.00 \\
\hline Watermelon & 10.27 & 7.17 & 12.29 & 4.83 & 8.65 & 6.20 & 5.96 & 2.00 \\
\hline Peppers & 8.63 & 15.17 & 17.88 & 20.53 & 15.77 & - & 22.15 & - \\
\hline Tomatoes & 8.84 & 14.61 & 12.90 & 11.36 & 13.33 & 18.26 & 18.05 & 13.55 \\
\hline Onion & 165.10 & 40.40 & 61.60 & 57.10 & 121.20 & 138.70 & 144.00 & 86.10 \\
\hline Head lettuces & 1.10 & 0.53 & 1.84 & 3.43 & 0.79 & 1.00 & 1.10 & 1.80 \\
\hline Sweet corn & 34.71 & 33.27 & 27.50 & 1.50 & 1.51 & 4.46 & 4.80 & 5.00 \\
\hline Pumkin & 57.55 & 182.25 & 376.53 & 255.45 & 283.54 & 218.99 & 236.74 & 464.05 \\
\hline Total & 270.83 & 424.59 & 565.32 & 433.54 & 406.58 & 380.50 & 356.79 & 593.51 \\
\hline
\end{tabular}

Table 5 Harvested area of most grown species of vegetables in the district of Nové Zámky in ha

\begin{tabular}{|l||c|c|c|c|c|c|c|c|c|}
\hline Vegetables & $\mathbf{2 0 0 2}$ & $\mathbf{2 0 0 3}$ & $\mathbf{2 0 0 4}$ & $\mathbf{2 0 0 5}$ & $\mathbf{2 0 0 6}$ & $\mathbf{2 0 0 7}$ & $\mathbf{2 0 0 8}$ & $\mathbf{2 0 0 9}$ & $\mathbf{2 0 1 0}$ \\
\hline \hline White cabbage & $\mathbf{7 4 . 6 0}$ & 58.86 & 80.91 & 43.71 & 75.26 & 63.01 & - & 40.95 & 35.40 \\
\hline Cauliflower & 110.36 & 133.57 & 117.57 & 114.89 & 135.55 & 81.59 & 67.69 & 55.93 & 116.24 \\
\hline Carrots & 24.52 & 45.09 & 17.56 & 9.72 & 14.85 & 12.59 & 9.76 & 10.51 & 10.31 \\
\hline Parsley and parsnips & 18.35 & 15.61 & 14.59 & 6.31 & 9.61 & 12.90 & 7.40 & 13.00 & 13.65 \\
\hline Gherkins & 36.54 & 14.31 & 17.38 & 15.44 & 20.35 & 11.96 & 14.17 & 15.32 & 10.60 \\
\hline Cucumbers & 13.48 & 6.47 & 10.46 & 12.39 & 9.92 & 13.37 & 11.91 & 11.51 & 10.11 \\
\hline Watermelon & 74.61 & 72.15 & 104.10 & 73.93 & 60.27 & 58.94 & 38.11 & 50.77 & 35.33 \\
\hline Peppers & 63.39 & 57.59 & 40.17 & 44.75 & 50.31 & 44.53 & 40.58 & 41.38 & 40.80 \\
\hline Tomatoes & 605.77 & 620.62 & 582.77 & 550.14 & 507.54 & 538.46 & 550.05 & 542.13 & 336.84 \\
\hline Onion & 20.34 & 46.95 & 57.91 & 8.53 & 15.47 & 18.32 & 22.16 & 32.51 & 43.20 \\
\hline Garlic & 14.89 & 13.36 & 17.65 & 17.63 & 16.63 & 16.50 & 16.10 & 14.58 & 14.57 \\
\hline Sweet corn & 4.36 & 13.04 & 7.92 & 3.15 & 2.22 & 4.75 & 7.75 & 6.06 & 26.52 \\
\hline Pumkin & - & 15.24 & 12.17 & 21.38 & 21.00 & 49.24 & 8.38 & 7.33 & 12.82 \\
\hline Total & $1,215.07$ & $1,274.70$ & $1,285.42$ & $1,048.63$ & $1,035.71$ & $1,006.69$ & 896.45 & 875.80 & 783.73 \\
\hline
\end{tabular}

Table 6 Production volume of most grown species of vegetables in the district of Komárno in $\mathrm{t}$

\begin{tabular}{|c|c|c|c|c|c|c|c|c|c|}
\hline Vegetables & 2002 & 2003 & 2004 & 2005 & 2006 & 2007 & 2008 & 2009 & 2010 \\
\hline White cabbage & $4,642.30$ & $6,730.40$ & $5,361.30$ & $1,299.50$ & 85.47 & $1,199.70$ & 989.50 & 794.80 & 450.50 \\
\hline Cauliflower & 397.70 & 329.60 & 583.30 & 387.10 & 511.00 & 306.00 & 191.80 & 190.20 & 117.60 \\
\hline Savoy cabbage & 485.40 & 587.10 & 505.90 & 237.20 & 467.90 & 239.00 & 124.20 & 336.70 & 158.50 \\
\hline Kohlrabi & 629.00 & 901.30 & 557.80 & 554.30 & 543.60 & 271.60 & 71.70 & 208.29 & 165.40 \\
\hline Carrots & $4,106.30$ & $2,044.60$ & $2,544.60$ & $1,733.50$ & $1,621.80$ & $1,470.10$ & $1,491.80$ & $1,202.60$ & 632.70 \\
\hline Parsley and parsnips & $2,941.90$ & 783.50 & $2,444.60$ & $1,786.00$ & $1,780.00$ & 726.60 & $2,250.30$ & $1,069.90$ & $1,026.80$ \\
\hline Cucumbers & 683.60 & 939.70 & 974.90 & $2,073.10$ & $2,306.70$ & 995.40 & 229.10 & 355.9 & 201.00 \\
\hline Watermelon & $2,296.40$ & $3,428.10$ & $1,799.60$ & $2,103.00$ & 619.80 & 777.30 & 753.70 & 542.90 & 52.30 \\
\hline Peppers & $2,914.40$ & $3,120.90$ & $2,273.70$ & $2,998.40$ & $2,566.90$ & $2,244.40$ & $1,368.30$ & $1,591.40$ & 629.10 \\
\hline Tomatoes & $7,291.60$ & $6,262.50$ & $5,108.20$ & $7,034.90$ & $6,428.60$ & $4,634.00$ & $3,569.90$ & $3,722.90$ & 226.30 \\
\hline Onion & $3,693.60$ & $1,222.00$ & $4,125.20$ & 963.40 & $1,412.70$ & $1,647.40$ & $1,770.70$ & 490.00 & 315.70 \\
\hline Head lettuces & 76.80 & 195.70 & 233.30 & 78.30 & 86.10 & 86.20 & 62.30 & 150.50 & 93.90 \\
\hline Peas & 10.60 & 14.20 & 98.10 & 10.60 & 10.60 & 1.00 & - & - & 85.50 \\
\hline Sweet corn & 47.80 & 8.10 & 68.30 & 61.4 & 27.70 & 9.70 & - & 25.70 & 57.80 \\
\hline Total & $32,369.80$ & $28,520.30$ & $29,383.10$ & $23,659.40$ & $22,563.70$ & $15,577.00$ & $13,638.20$ & $11,510.30$ & $4,986.30$ \\
\hline
\end{tabular}


Table 7 Production volume of most grown species of vegetables in the district of Levice in $t$

\begin{tabular}{|c|c|c|c|c|c|c|c|c|c|}
\hline Vegetables & 2002 & 2003 & 2004 & 2005 & 2006 & 2007 & 2008 & 2009 & 2010 \\
\hline White cabbage & $1,327.70$ & $2,634.20$ & - & 251.50 & $1,037.60$ & 252.70 & - & 40.60 & 86.20 \\
\hline Carrots & 420.70 & $2,650.60$ & $1,190.40$ & 647.50 & 715.20 & 164.20 & 274.80 & 166.00 & 482.50 \\
\hline Parsley and parsnips & 255.50 & 30.10 & 125.30 & 91.50 & 102.60 & 47.20 & 58.60 & 97.00 & 126.30 \\
\hline Gherkins & $2,130.30$ & $1,549.10$ & 698.40 & $2,106.10$ & $1,701.60$ & 799.60 & 340.30 & 408.20 & 491.70 \\
\hline Cucumbers & 45.10 & 200.80 & 95.80 & 163.90 & 56.60 & 12.30 & 6.40 & 6.10 & 120.00 \\
\hline Peppers & $1,649.60$ & $1,387.50$ & $1,257.50$ & $1,308.50$ & $1,357.70$ & 964.60 & 154.70 & 230.30 & 105.40 \\
\hline Tomatoes & $5,839.40$ & $6,102.20$ & $8,157.20$ & $3,645.70$ & $4,327.20$ & $1,110.60$ & 571.10 & 167.60 & 540.10 \\
\hline Onion & $1,570.40$ & 420.30 & $1,702.60$ & 847.60 & 722.80 & 233.10 & $1,373.20$ & 494.00 & $1,353.60$ \\
\hline Peas & 191.90 & 121.50 & 600.20 & 913.10 & 5.4 & - & - & - & $1,181.40$ \\
\hline Sweet corn & $2,255.10$ & $2,313.90$ & $1,425.30$ & 803.60 & 234.70 & 896.40 & $3,120.30$ & $1,127.70$ & $2,680.70$ \\
\hline Pumkin & - & 71.70 & 146.50 & - & 248.50 & 12.30 & 161.50 & 130.60 & 145.10 \\
\hline Total & $18,062.90$ & $19,798.20$ & $18,509.80$ & $12,238.80$ & $11,801.90$ & $6,042.70$ & $7,490.80$ & $5,023.20$ & $8,275.20$ \\
\hline
\end{tabular}

Table 8 Production volume of most grown species of vegetables in the district of Nitra in t

\begin{tabular}{|c|c|c|c|c|c|c|c|c|c|}
\hline Vegetables & 2002 & 2003 & 2004 & 2005 & 2006 & 2007 & 2008 & 2009 & 2010 \\
\hline White cabbage & 875.00 & $1,392.20$ & $2,393.90$ & 787.20 & 942.00 & 615.00 & $1,264.50$ & $1,000.80$ & $1,194.80$ \\
\hline Cauliflower & 75.00 & 107.20 & 186.70 & 160.10 & 457.60 & 93.10 & 81.00 & 106.80 & 71.30 \\
\hline Carrots & 61.40 & 36.90 & 27.20 & 102.30 & 70.30 & 38.90 & 199.70 & 193.50 & 58.30 \\
\hline Gherkins & 706.30 & 524.50 & 227.90 & 316.30 & 472.10 & 99.00 & 190.70 & 105.30 & 85.90 \\
\hline Cucumbers & 20.60 & 30.30 & 23.60 & 39.60 & 47.70 & 63.30 & 236.30 & 295.50 & 295.00 \\
\hline Watermelon & 88.80 & 217.90 & 70.80 & 249.90 & 44.70 & 66.10 & 61.40 & 86.30 & 5.00 \\
\hline Peppers & 29.20 & 141.00 & 457.90 & 368.10 & 620.80 & 738.70 & - & 377.30 & - \\
\hline Tomatoes & 105.20 & 272.70 & 749.80 & 730.30 & 859.90 & $1,196.90$ & $1,488.50$ & 729.30 & 76.30 \\
\hline Onion & 165.10 & 40.40 & 61.60 & 57.10 & 121.20 & 138.70 & 144.00 & 86.10 & - \\
\hline Head lettuces & - & 23.30 & 5.00 & 47.20 & 60.50 & 1.90 & 8.50 & 5.50 & 21.30 \\
\hline Sweet corn & 286.30 & 267.00 & 329.70 & 325.70 & 6.00 & 8.90 & 43.40 & 29.70 & 23.00 \\
\hline Pumkin & - & 744.20 & 467.00 & $4,686.70$ & 378.50 & 248.30 & 202.40 & 296.20 & 260.50 \\
\hline Total & $2,939.30$ & $4,191.60$ & $5,432.50$ & $8,431.00$ & $4,413.70$ & $3,428.60$ & $4,948.80$ & $3,323.80$ & $2,255.80$ \\
\hline
\end{tabular}

125 ha. From that time until 2010, the area for many species significantly decreased or ceased to exist (Table 3 ).

In the district of Nitra, the total acreage of vegetables increased from 271 ha to 594 ha between 2003 and 2010. The increase in growth areas was recorded in pumpkins, cucumbers and carrots. For other species, the growing area varied and in some cases also decreased during those years (Table 4).

The Nové Zámky district is characterized by the production of tomatoes and cauliflowers (Table 5). In 2002, tomatoes were grown on 606 hectares and in 2010 on the area of 337 ha. Cauliflower areas reached their maximum in 2006 (136 ha), whereas the minimum in 2009 (Ďurovičová et al, 2003).

\section{The production of vegetables}

In the past, the district of Komárno ranked first in production of vegetables in the Nitra region. In 2002, the district produced a total of 32,370 t of vegetables, but by 2010 , this amount dropped to 4,538 $\mathrm{t}$ (Rakovská et al., 2009). Most of the production represented tomatoes $(7,291 \mathrm{t})$, but in 2010, their production was only $226 \mathrm{t}$. Similarly, the production of white cabbage, carrots, onions, parsley, peppers and watermelons decreased (Table. 6).

The total production of vegetables in the district of Levice decreased between 2002 and 2010 from 18,063 t to 8,275 t. Similar pattern was also observed for other types of vegetables. Currently, the production of sweet corn (2,681 tons) and onion (1,354 t) dominate. The production of tomatoes, gherkins and carrots reach about $500 \mathrm{t}$ each (Table 7).

In the district of Nitra, the production of white cabbage $(1,195 \mathrm{t})$, cucumbers $(295 \mathrm{t})$ and pumpkins (261 t) prevailed in 2010. The critical decline in production was recorded in gherkins and sweet corn. Conversely, a significant increase occurred in cucumbers (Table 8). The overall decline for all 
Table 9 Production volume of most grown species of vegetables in the district of Nové Zámky (t)

\begin{tabular}{|c|c|c|c|c|c|c|c|c|c|}
\hline Vegetables & 2002 & 2003 & 2004 & 2005 & 2006 & 2007 & 2008 & 2009 & 2010 \\
\hline White cabbage & 957.60 & $1,256.00$ & $1,729.40$ & 716.40 & $1,868.00$ & $1,486.30$ & - & 731.80 & 333.30 \\
\hline Cauliflower & 679.40 & $1,592.00$ & 161.50 & $1,001.40$ & $1,130.60$ & $1,016.00$ & 572.50 & 338.80 & 400.30 \\
\hline Carrots & 343.30 & 788.50 & 380.00 & 169.50 & 412.70 & 181.10 & 158.20 & 197.60 & 112.00 \\
\hline Parsley and parsnips & 70.90 & 68.10 & 90.10 & 58.20 & 71.70 & 38.70 & 21.90 & 198.50 & 62.60 \\
\hline Gherkins & 408.40 & 172.90 & 187.70 & 285.60 & 418.50 & 76.90 & 126.70 & 202.50 & 56.80 \\
\hline Cucumbers & 352.20 & 282.70 & 579.10 & 464.10 & 107.50 & 454.90 & $1,265.80$ & $1,116.10$ & 892.40 \\
\hline Watermelon & 492.40 & $1,445.10$ & 928.50 & $1,108.30$ & 732.20 & 743.90 & 200.10 & 213.80 & 144.00 \\
\hline Peppers & 513.90 & 379.60 & 356.40 & 498.00 & 566.10 & 437.80 & 353.50 & 386.60 & 188.10 \\
\hline Tomatoes & $13,087.30$ & $8,493.70$ & $16,037.80$ & $14,839.80$ & $15,833.00$ & $14,698.30$ & $18,867.90$ & $15,345.20$ & $4,450.60$ \\
\hline Onion & 241.20 & 305.30 & 596.80 & 111.30 & 147.30 & 147.90 & 288.40 & 318.10 & 142.40 \\
\hline Garlic & 73.70 & 66.20 & 53.00 & 52.90 & 54.90 & 65.90 & 51.90 & 46.20 & 78.30 \\
\hline Sweet corn & 26.70 & 73.20 & 57.70 & 14.80 & 13.70 & 18.30 & 45.00 & 34.40 & 96.30 \\
\hline Pumkin & - & 54.00 & 15.80 & 28.20 & 30.30 & 26.20 & 6.60 & 14.90 & 44.10 \\
\hline Total & $18,147.00$ & $16,023.90$ & $23,889.40$ & $20,233.60$ & $21,919.50$ & $19,750.30$ & $23,752.10$ & $19,525.90$ & $7,261.50$ \\
\hline
\end{tabular}

over the vegetable production represents 23\% (Valšíková and Ryban, 2012).

The production of vegetables in the district of Nové Zámky visibly changed between 2002 and 2009. In 2010, the harvest was lower by $63 \%$ compared to the previous year. This decrease was mainly caused by a dramatic fall in production of tomatoes and cabbage to one-third (Table 9).

\section{Summary}

The Nitra Region is the most productive area in terms of vegetable production in Slovakia. Vegetables are grown at the largest area of arable land ( 937 ha) in the district of Levice. Vegetables in the districts of Komárno and Nové Zámky were planted on the area of 780 ha. The smallest size of vegetable areas was recorded in the districts of Topolčany and Zlaté Moravce. The greatest volume of vegetables was produced in the district of Levice (7,343 t) and Nové Zámky (7,114 t). Less vegetables were produced in the districts of Komárno $(4,538 \mathrm{t})$, Šal'a $(2,775 \mathrm{t})$ and Nitra $(2,091 \mathrm{t})$. When comparing the vegetable production between 2002 and 2010, it can be concluded that it declined in all districts. Among the most grown vegetables we can include cabbage, carrot, parsley, onion, tomato, pepper, watermelon and sweet corn.

ĎUROVIČOVÁ, M. 2003. Definitívne údaje o úrode polnohospodárskych plodín a zeleniny v Nitrianskom kraji. ŠÚ SR, pracovisko v Nitre, 51 Tab.

\section{References}

JUREKOVÁ, Z. a i. 2005. Slovenské záhradníctvo. Trendy vedy, praxe a vysokoškolského vzdelávania. Nitra : SPU, 2005. 216 s. ISBN 80-8069-629-2.

MERAVÁ, E. a i. 2009. Zelenina - situačná a výhl'adová správa k 31.12 2008. Bratislava : VÚEPP, 2009. 57 s. ISBN 978-80-8058-487-0.
MERAVÁ, E. a i. 2011. Zelenina - situačná a výhladová správa k 31.12.2010. Bratislava : VÚEPP, MPaRV SR, 2011. 54 s. ISBN 978-80-8058-562-4.

RAKOVSKÁ, E. 2009. Definitívne údaje o úrode pol'nohospodárskych plodín a zeleniny v Nitrianskom kraji za rok 2008. ŠÚ SR, pracovisko v Nitre, $51 \mathrm{Tab}$.

ROZBORILOVÁ, E. 2010. Definitívne údaje o úrode pol'nohospodárskych plodín a zeleniny v SR za rok 2010. Bratislava : Štatistický úrad SR. 2010. ISBN 748-80-8121-268-0.

SRNIČKOVÁ, K. 2012. Zeleninárska produkcia v Nitrianskom kraji. Diplomová práca, Nitra : SPU, 2012.96 s.

UHER, A. 2011. Zelenina vo výžive a jej rizikové faktory. 2. vydanie, Nitra : SPU, 2011. 65 s. ISBN 978-80-552-0518-2.

VALŠÍKOVÁ, M. - UHER, A. 2009. Situácia v zeleninárskom sektore z pohl'adu vedy, výskumu a výroby v Slovenskej republike. In: Acta horticulturae et regiotecturae, 2009. mimoriadne číslo, s. 232-237. ISSN 1335-2563.

VALŠÍKOVÁ, M. - RYBAN, R. 2012. Produkcia mrkvy obyčajnej v SR In: Zahradnictví, roč. 10, 2012. č. 5, s. 23-26. ISSN 1213-7596.

VALŠíKOVÁ, M. - STANO, J. - KOREŇOVÁ, M. - ZEMAN. S. 2011. Vegetable production and consumption in the Slovak Republic. Výživa a zdravie. Nitra : SPU. Zborník na CD ROM. ISBN 978-80-552-0699-9.

Contact address:

prof. Ing. Magdaléna Valšíková, PhD., Slovak University of Agriculture in Nitra, Horticulture and Landscape Engineering Faculty, Department of Vegetables-Production. Tr. Andreja Hlinku 2, 94976 Nitra, Slovak Republic, phone: +421/37 641 42 26, e-mail: Magdalena.Valsikova@uniag.sk 